JOURNAL of

MAINE MEDICAL CENTER Journal of Maine Medical Center

\title{
Spouses with Pulmonary Mycobacterium Avium Complex Infections: A Case Report
}

\author{
Gregg Raymond MD \\ Maine Medical Center, Portland, Maine
}

Et al.

Follow this and additional works at: https://knowledgeconnection.mainehealth.org/jmmc

Part of the Infectious Disease Commons

\section{Recommended Citation}

Raymond, Gregg MD and Wood, Emily MD (2021) "Spouses with Pulmonary Mycobacterium Avium Complex Infections: A Case Report," Journal of Maine Medical Center. Vol. 3 : Iss. 2 , Article 12.

Available at: https://knowledgeconnection.mainehealth.org/jmmc/vol3/iss2/12 https://doi.org/10.46804/ 2641-2225.1077

The views and thoughts expressed in this manuscript belong solely to the author[s] and do not reflect the opinions of the Journal of Maine Medical Center or MaineHealth.

This Case Report is brought to you for free and open access by Maine Medical Center Department of Medical Education. It has been accepted for inclusion in the Journal of Maine Medical Center by an authorized editor of the MaineHealth Knowledge Connection. For more information, please contact Dina McKelvy mckeld1@mmc.org.

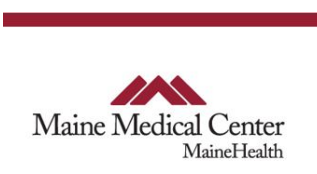




\section{Spouses with Pulmonary Mycobacterium Avium Complex Infections: A Case Report}

\section{Acknowledgements}

We would like to thank Kathleen Fairfield MD, MPH, DrPH for her help in the preparation of this manuscript.

\section{Authors}

Gregg Raymond MD and Emily Wood MD 


\title{
Spouses with Pulmonary Mycobacterium Avium Complex Infections: A Case Report
}

\author{
Gregg Raymond, MD, ${ }^{1}$ Emily Wood, $\mathrm{MD}^{1,2}$ \\ ${ }^{1}$ Department of Medicine, Maine Medical Center, Portland, ME, '2Division of Infectious Diseases, Maine Medical Center, \\ Portland, ME
}
Introduction: Mycobacterium avium complex (MAC) is the most common cause of pulmonary infections due to nontuberculous mycobacterium in the United States. While there have been reports of geographic variations in incidence as well as disease clusters among genetically related individuals, there have been no published reports describing clusters of co-occurring pulmonary MAC disease among genetically unrelated individuals.

Clinical Findings: Patient 1 was a 56 -year-old male with worsening asthma exacerbations associated with recurrent
bronchitis. Patient 2 was a 55 -year-old female with worsening asthma exacerbations, progressive
dyspnea, and recurrent bronchitis. The 2 patients lived together, were immunocompetent, and
genetically unrelated. They were frequently exposed to aerosolized soil and inadequately heated water.

Clinical Course: Patient 1 had normal imaging and his sputum grew out MAC. He responded quickly to treatment with azithromycin, rifampin, and ethambutol. Patient 2 had computed tomographic imaging that revealed tree-in-bud and ground-glass opacities. Her sputum samples did not grow out acid-fast bacillus; however, 14 months after Patient 1 was diagnosed, her bronchoscopy samples ultimately grew out MAC. Patient 2 clinically responded quickly to the same treatment as Patient 1 , with resolution of her dyspnea, bronchitis, and radiographic abnormalities.

Conclusions: We describe 2 cohabitating, genetically unrelated, immunocompetent patients with pulmonary MAC infections. The likely cause of these infections was a common exposure to aerosolized soil and inadequately heated water. We report the unusual co-occurrence of pulmonary MAC among genetically unrelated individuals living together.

Keywords: pulmonary Mycobacteria avium complex, nontuberculosis mycobacterium, immunocompetent, case reports, disease cluster

\section{CASE PRESENTATION}

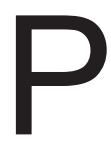
atient 1 was a 56-year-old male with previously well-controlled asthma. He had an 8-year history of worsening asthma exacerbations associated with recurrent mucopurulent bronchitis that had responded temporarily to multiple short courses of azithromycin. His physical exam revealed scattered wheezes and rhonchi. His physicians were reassured by normal imaging and, for several years, focused their care on optimizing his asthma control. Once the diagnosis of Mycobacterium avium

Correspondence: Gregg Raymond, MD

Department of Medicine, Maine Medical Center

22 Bramhall St. Portland, ME 04102

raymog@mmc.org complex (MAC) was considered, 3 consecutive sputum mycobacterial cultures collected from the patient grew out pan-susceptible MAC. Patient 1 responded quickly to treatment with azithromycin (500 mg), rifampin (600 mg), and ethambutol (1200 mg) 3 times weekly. After 6 weeks of treatment, he had persistent negative cultures and improved asthma control. He completed 14 months of antimicrobial treatment.

Patient 2 was a 55-year-old female with previously well-controlled asthma. She had a 4-year history of recurrent mucopurulent bronchitis, worsening asthma exacerbations, and progressive dyspnea that had responded temporarily to short courses of azithromycin. After her husband was diagnosed 
with pulmonary MAC, she approached her physicians about the possibility that she could have a MAC infection too. They felt that this diagnosis was unlikely, but they collected several sputum samples from the patient and sent them for mycobacterial culture. The results of these cultures were all negative. Her subsequent care focused unsuccessfully on improving her asthma control, and she had steadily worsening dyspnea. Her physical exam revealed mild respiratory distress and scattered rhonchi and wheezes. CT (computed tomographic) imaging obtained over 1 year showed progressive bronchial wall thickening, bronchiectasis, peripheral tree-in-bud opacities, and confluent areas of ground-glass opacities. Due to these radiographic abnormalities, the possibility of pulmonary MAC was reconsidered, as well as an infection with Nocardia or Actinomyces. Cultures obtained from her initial bronchoscopy did not grow out any clear pathogen. Because of her clinical deterioration and worsening CT findings, a second bronchoscopy was performed 6 months later. Pan-susceptible MAC grew out from the second bronchoscopy samples about 14 months after Patient 1 was diagnosed. Patient 2 responded within weeks to treatment with azithromycin (500 $\mathrm{mg}$ ), rifampin (600 mg), and ethambutol (1200 mg) 3 times weekly, with resolution of her dyspnea and mucopurulent bronchitis. Her asthma became easy to control. CT Imaging 10 months after treatment initiation showed complete resolution of the tree-inbud and ground-glass opacities. She completed 12 months of antimicrobial treatment. Her final sputum mycobacterial cultures are pending.

Patient 1 and 2 were genetically unrelated, married, and living together. Both patients had never smoked and neither had a prior personal or family history of recurrent infections. Both patients had negative evaluations for immunodeficiency, which included normal lymphocyte subsets on flow cytometry, normal antibody titers to pneumococcal vaccination, and normal immunoglobulin levels. They were avid gardeners who were frequently exposed to aerosolized soil. Prior to their MAC diagnoses, their water heater had been set to a very low temperature. Their water was obtained from a municipal water supply. Genotyping to compare strains from Patient 1 and Patient 2 was not available, and their home water system was not tested for the presence of MAC. The patients had 2 children who lived with them, but who had https://knowledgeconnection.mainehealth.org/jmmc/vol3/iss2/12 DOI: $10.46804 / 2641-2225.1077$ never developed any pulmonary symptoms. Their children showered less frequently than their parents and were not exposed to aerosolized soil.

\section{DISCUSSION}

Nontuberculosis mycobacterium (NTM) are species of microbes in the genus Mycobacterium that are not part of the Mycobacterium tuberculosis complex or Mycobacterium leprae. MAC includes at least 2 species, Mycobacterium avium and Mycobacterium intracellulare, and is the most common cause of pulmonary infections due to NTM in the United States. MAC is a ubiquitous organism and readily isolated from the environment, particularly in water and soil. ${ }^{1,2}$ MAC can be aerosolized from water sources. Studies have shown that environmental and clinical isolates can be phenotypically the same, suggesting an environmental source of infection. ${ }^{3}$ In addition, inadequately heated water can promote the growth of MAC in home water systems, likely due to the temperature sensitivity of these organisms. ${ }^{4}$ Person-to-person transmission of MAC is not believed to occur, but there have been reports of MAC disease clusters among genetically related individuals. ${ }^{5}$ Geographic variations in the incidence of MAC-related disease have been found. . $, 7,8$ but regions with disease clusters have not been reported in New England, where our patients resided. Our review of the medical literature did not reveal any reports of pulmonary MAC disease clusters among genetically unrelated patients living together.

In this case report, we describe 2 genetically unrelated, immunocompetent patients who lived together and developed pulmonary MAC infections. In both patients, the diagnosis of pulmonary MAC was made only after many years of treatment by their physicians. These patients both showered with inadequately heated water and were also exposed to aerosolized soil. Their inadequately heated water may have increased the likelihood that MAC would have been present in their showering water. It is possible that MAC was transmitted from Patient 1 to Patient 2; however, genotyping to compare the cultured isolates from our patients was not available to investigate this hypothesis. Given that MAC has not been shown to spread between humans, the likely cause of our patients' infections was a common exposure to aerosolized water or soil that contained MAC. Our patients' children did not develop pulmonary symptoms, which may be 
due to less exposure to aerosolized water, lack of exposure to aerosolized soil, and potentially increased resistance to MAC infections due to their younger age.

A major limitation of this case report was that we were not able to draw more definitive conclusions about the source of our patients' infections. Cultures were not obtained from our patients' shower heads, hot water tank, or soil to demonstrate that MAC was present. Also, genotyping was not available to check for concordance between our patients' MAC isolates and potential environmental isolates.

This case report presents an unusual co-occurrence of pulmonary MAC between genetically unrelated individuals living together. These infections were likely related to shared environmental exposures to MAC. This case report also serves as a reminder to clinicians to consider MAC infections in the differential diagnosis when evaluating patients with worsening pulmonary symptoms of unclear etiology.

\section{Conflict of Interest: None}

Acknowledgements: We would like to thank Kathleen Fairfield, MD, MPH, DrPH for her help in the preparation of this manuscript.

\section{REFERENCES}

1. Lande L, Alexander DC, Wallace RJ Jr, et al. Mycobacterium avium in community and household water, Suburban Philadelphia, Pennsylvania, USA, 2010-2012. Emerg Infect Dis. 2019;25(3): 473-481. doi:10.3201/eid2503.180336

2. Nishiuchi Y, Iwamoto T, Maruyama F. Infection sources of a common mon-tuberculous mycobacterial pathogen, Mycobacterium avium complex. Front Med (Lausanne). 2017;4:27. doi:10.3389/ fmed.2017.00027

3. Nishiuchi Y, Maekura R, Kitada S, et al. The recovery of Mycobacterium avium-intracellulare complex (MAC) from the residential bathrooms of patients with pulmonary MAC. Clin Infect Dis. 2007;45(3):347-351. doi:10.1086/519383

4. Falkinham JO 3rd. Nontuberculous mycobacteria from household plumbing of patients with nontuberculous mycobacteria disease. Emerg Infect Dis. 2011;17(3):419-424. doi: 10.3201/ eid1703.101510

5. Colombo RE, Hill SC, Claypool RJ, Holland SM, Olivier KN. Familial clustering of pulmonary nontuberculous mycobacterial disease. Chest. 2010;137(3): 629-634. doi: 10.1378/chest.09-1173

6. Adjemian J, Olivier KN, Seitz AE, Falkinham JO 3rd, Holland SM, Prevots DR. Spatial clusters of nontuberculous mycobacterial lung disease in the United States. Am J Respir Crit Care Med. 2012;186(6):553-558. doi:10.1164/rccm.201205-0913OC

7. Mirsaeidi M, Vu A, Leitman P, et al. A patient-based analysis of the geographic distribution of Mycobacterium avium complex, Mycobacterium abscessus, and Mycobacterium kansasii Infections in the United States. Chest. 2017;151(4):947-950. doi:10.1016/j. chest.2017.02.013

8. Spaulding AB, Lai YL, Zelazny AM, et al. Geographic Distribution of nontuberculous mycobacterial species identified among clinical isolates in the United States, 2009-2013. Ann Am Thorac Soc. 2017;14(11):1655-1661. doi:10.1513/AnnalsATS.201611-860OC 